\title{
Control and Implementation of 2-DOF Lower Limb Exoskeleton Experiment Platform
}

\author{
Zhenlei Chen', Qing Guo ${ }^{1,2^{*}} \mathbb{0}$, Huiyu Xiong ${ }^{3}$, Dan Jiang ${ }^{4}$ and Yao Yan ${ }^{1,2}$
}

\begin{abstract}
In this study, a humanoid prototype of 2-DOF (degrees of freedom) lower limb exoskeleton is introduced to evaluate the wearable comfortable effect between person and exoskeleton. To improve the detection accuracy of the humanrobot interaction torque, a BPNN (backpropagation neural networks) is proposed to estimate this interaction force and to compensate for the measurement error of the 3D-force/torque sensor. Meanwhile, the backstepping controller is designed to realize the exoskeleton's passive position control, which means that the person passively adapts to the exoskeleton. On the other hand, a variable admittance controller is used to implement the exoskeleton's active followup control, which means that the person's motion is motivated by his/her intention and the exoskeleton control tries best to improve the human-robot wearable comfortable performance. To improve the wearable comfortable effect, serval regular gait tasks with different admittance parameters and step frequencies are statistically performed to obtain the optimal admittance control parameters. Finally, the BPNN compensation algorithm and two controllers are verified by the experimental exoskeleton prototype with human-robot cooperative motion.
\end{abstract}

Keywords: Lower limb exoskeleton, BP neural network, Backstepping controller, Variable admittance strategy

\section{Introduction}

In recent decades, the wearable robot has been widely applied in the field of medical rehabilitation engineering. One typical representative is the lower limb exoskeleton, which has played an essential role in the patient's rehabilitation training with limb injuries and hemiplegia.

Generally, several exoskeleton prototypes have been developed for various functional requirements. Khamar et al. [1] designed the knee exoskeleton composed of a four-bar mechanism and a curved one. Aguirre-Ollinger et al. [2] developed a stationary 1-DOF exoskeleton to assist the knee with flexion and extension exercises. Furthermore, the lower limb exoskeleton was driven by a hydraulic actuator and series elastic actuators (SEAs) in Refs. [3] and [4], respectively.

The exoskeleton is a type of wearable robot attached to the operator's body and helps the operator complete

\footnotetext{
*Correspondence: guoqinguestc@uestc.edu.cn

${ }^{1}$ School of Aeronautics and Astronautics, University of Electronic Science

and Technology of China, Sichuan 611731, Chengdu, China

Full list of author information is available at the end of the article
}

specific tasks [5]. It is worth noting that the system stability of human-robot coordinated motion and the motion tracking the designed controller's performance are crucial, which directly determines the wearable comfortable effect between person and exoskeleton. Hence, a repetitive learning controller was adopted for motion assistance in Ref. [3]. An adaptive control scheme and a learning control approach were proposed to handle periodic uncertainties in Ref. [6]. Guo et al. [7, 8] synthesized the structural parameter of an overload carrying exoskeleton. They proposed a human-robot coordinated control approach to improve the exoskeleton's aid-force efficiency in the walking stance phase. Li et al. [9] presented a fuzzy approximation based adaptive backstepping controller in the exoskeleton.

Furthermore, with the development of artificial intelligence (AI) technology, some AI algorithms have also been used in the exoskeleton's control design. For example, Duong et al. [10] proposed an adaptive controller based on the Radial Basis Function (RBF) neural network to compensate for the dynamic uncertainty error. Meanwhile, He et al. [11] designed an adaptive neural 
network controller with input saturation to improve the upper limb exoskeleton's dynamic performance.

According to Ref. [12], the control mode of the exoskeleton is roughly divided into two categories: active mode and passive mode. For the passive mode, the desired trajectories of exoskeleton are designed based on specific rehabilitation training tasks in advance. Comparing with the exoskeleton with passive mode, the desired trajectory of active mode is generated based on operator's real-time motion instead of setting in advance.

The real-time human motion intention can be obtained according to the human-robot interaction, divided into the cognitive human-robot interaction (cHRI) and the physical human-robot interaction (pHRI). Since the human-robot interaction force/torque belongs to the pHRI, both admittance control and impedance control [13] effectively address the human-robot coordinated motion. As introduced in Ref. [14], some excellent literature about admittance control and impedance control of human-robot coordinated motion has been published. For example, an admittance control based on human stiffness estimator was adopted for the exoskeleton robot, and a variable admittance control based on online neural network training is proposed in Ref. [15]. Buchli et al. [16] presented a learning variable impedance control with a reinforcement learning (RL) algorithm $\mathrm{PI}^{2}$ to handle some uncertainties. Yang et al. [17] adopted an admittance adaptation method in robots to interact with unknown environments. Additionally, some literature has also discussed the stability of admittance control and impedance. Furthermore, the detection method of admittance controller stability and the corresponding admittance parameter updating based on the energy tank was proposed [18-20]. Meanwhile, the frequency domain detection for admittance controller stability was given in Refs. [21, 22]. Furthermore, a stability condition related to stiffness, damping, and their rates of change was proposed in Ref. [23]. Kim et al. [24] presented an admittance controller based on the passivity theory for a powered upper-limb exoskeleton to guarantee humanrobot stability. Gui et al. [25] presented a practical and adaptive method to estimate active joint torque using electromyography (EMG) signals for a custom lower limb robotic exoskeleton with two DOFs. Zhuang et al. [26] presented an EMG-based admittance controller to realize both synchronized and stable human-robot interaction. Yu et al. [27] presented an adaptive impedance control strategy to track a target impedance model and neural networks are used to compensate for uncertainties in robotic dynamics. The compliant control has also been applied to some other field, such as collaborative robot [28] and leg robot [29].
In this study, a humanoid prototype of the 2-DOF lower limb exoskeleton was designed, and two exoskeleton control schemes are introduced to realize the human-robot coordinated motion. Inspired by previous studies about the online instability index $[21,22]$, the exoskeleton's variable admittance control is studied to improve the wearable comfortable effect between person and exoskeleton. The main contributions of this paper are twofold.

(I) The 2-DOF lower limb exoskeleton prototype is designed based on a rigid humanoid structure. Meanwhile, the human-robot interaction force is measured by the 3-D force/torque sensor. To compensate for this sensor measurement error, the BP neural network can online estimate the real interaction force.

(II) Two modes realize the human-robot coordinated motion. In the passive exoskeleton mode, the backstepping controller is designed to realize the exoskeleton's desirable position tracking. Meanwhile, the person should passively adapt to the exoskeleton and endure a certain human-robot impedance. In the exoskeleton active mode, a variable admittance controller is used to implement the exoskeleton's follow-up control. In this condition, the person's motion is motivated by his/ her intention, and then the exoskeleton is driven by the admittance controller to improve the human-robot wearable comfortable performance. The variable admittance controller is designed according to the experimental statistical method. Finally, the effectiveness of two motion modes has been verified by the wearable experiment of the specified tester as shown in Figure 1.

The remainder of this paper is organized as follows. The mechanical structure of the exoskeleton prototype and the human-robot interaction force estimation is introduced in Section 2. The backstepping controller and admittance controller in the passive mode and active mode is designed in Section 3. The variable admittance parameter strategy based on the statistical experiment results is designed in Section 4. The experimental verification is given in Section 5. Finally, the conclusions are drawn in Section 6.

\section{Design of the Exoskeleton Platform}

\subsection{Anatomy of Human Upper-limb}

The humanoid mechanical structure of the exoskeleton prototype is shown in Figure 2. To guarantee strength and durability, the exoskeleton limbs are manufactured of stainless steel material. The prototype leg has 2-DOF motion, i.e., the hip and knee joints. Both joints are driven by a disc motor with a servo driving module. Several sensors and elements have been installed between person and robot, which may decline the compactness and coupling effect of the human-robot system. A curved rigid structure is designed to replace the 


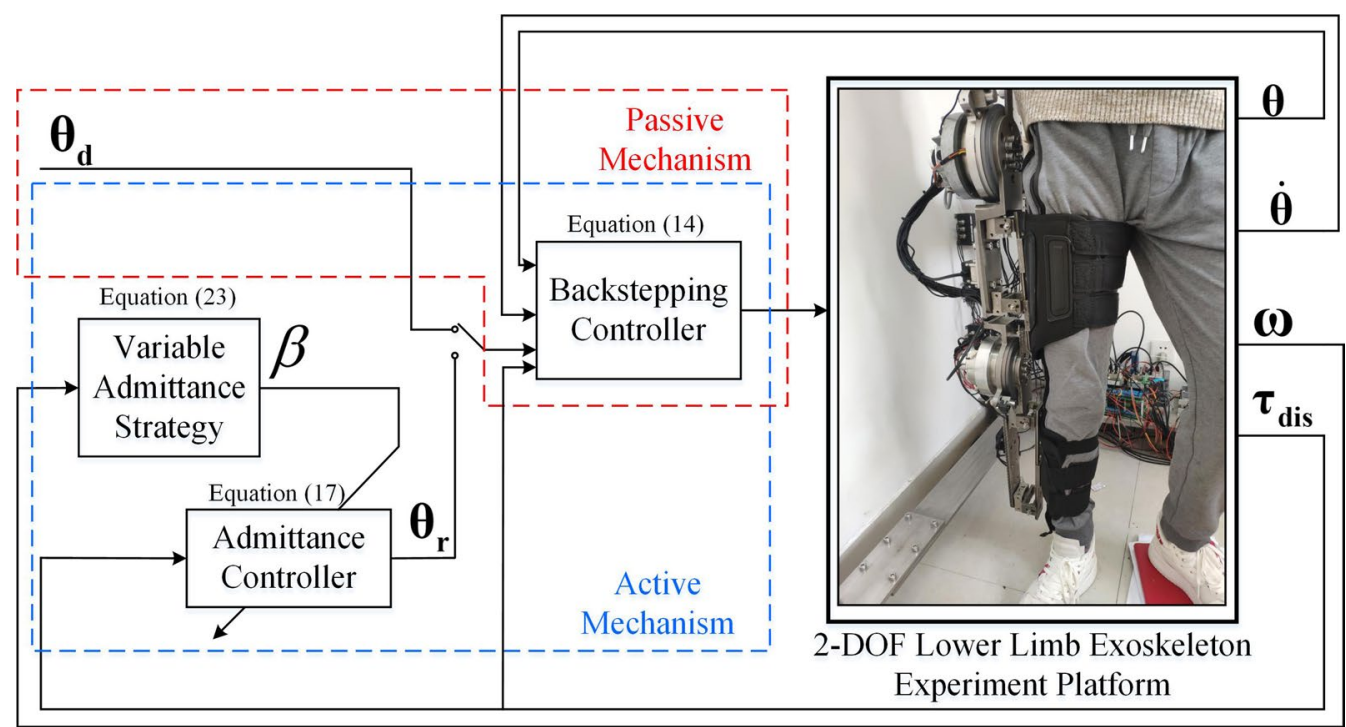

Figure 1 Control scheme of lower limb exoskeleton in active model and passive mode
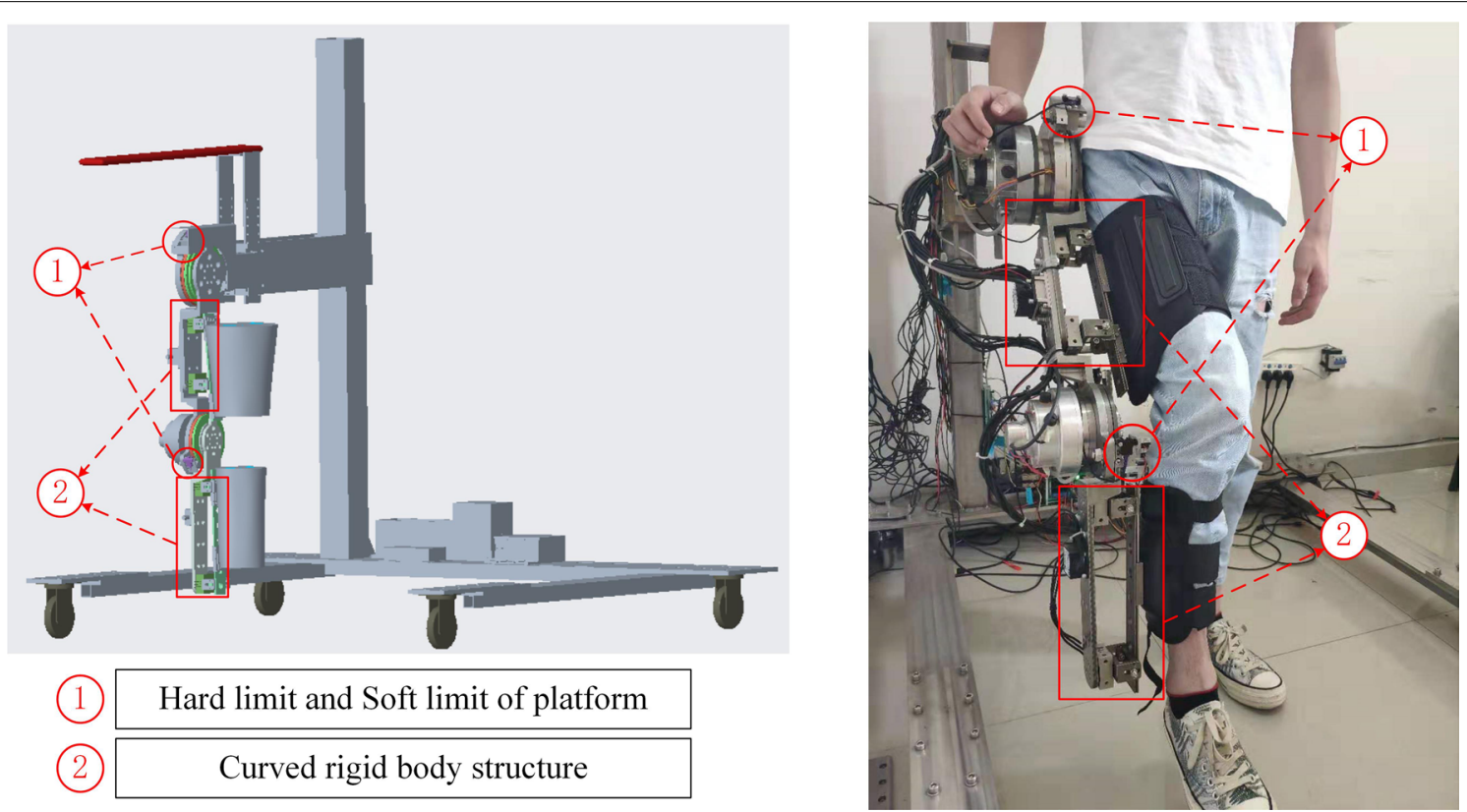

Figure 2 Mechanical structure of the 2-DOF lower limb exoskeleton platform

traditional straight thigh and shank of the exoskeleton to solve this problem. Furthermore, both the thigh and the shank length can be regulated concerning the different tester.

To protect the operator's safe, analyzing the physiological structure of the human lower limb, two types of limited position protection by mechanical and photoelectric devices are adopted. The exoskeleton is equipped with several universal wheels to move/fixed the mechanical frame. Meanwhile, the exoskeleton prototype height can be regulated in practice to suit the different tester.

\subsection{Electrical System Design}

The electrical system is also the critical component of the exoskeleton prototype, which includes the realtime data collection and processing, the control commands distribution, and the servo motor actuator. 
Hence, the electrical system is classified as three parts: the data collection module, the actuator driving module, and the control module, as shown in Figures 3, 4. The data collection module includes the human-robot interaction forces measured by 3-D force/torque sensors (JNSH-2-10kg-BSQ-12), two joint angles measured by the absolute encoder (INC-4-150 and INC-3-125), and the driven torque measured by the torque sensor integrated into the motor. To drive the leg of exoskeleton motion, the actuator driving module executes the control demands by two servo motor actuators (GDM1$100 \mathrm{~N} 2 / 120 \mathrm{~N} 2)$ together with two servo motor drivers

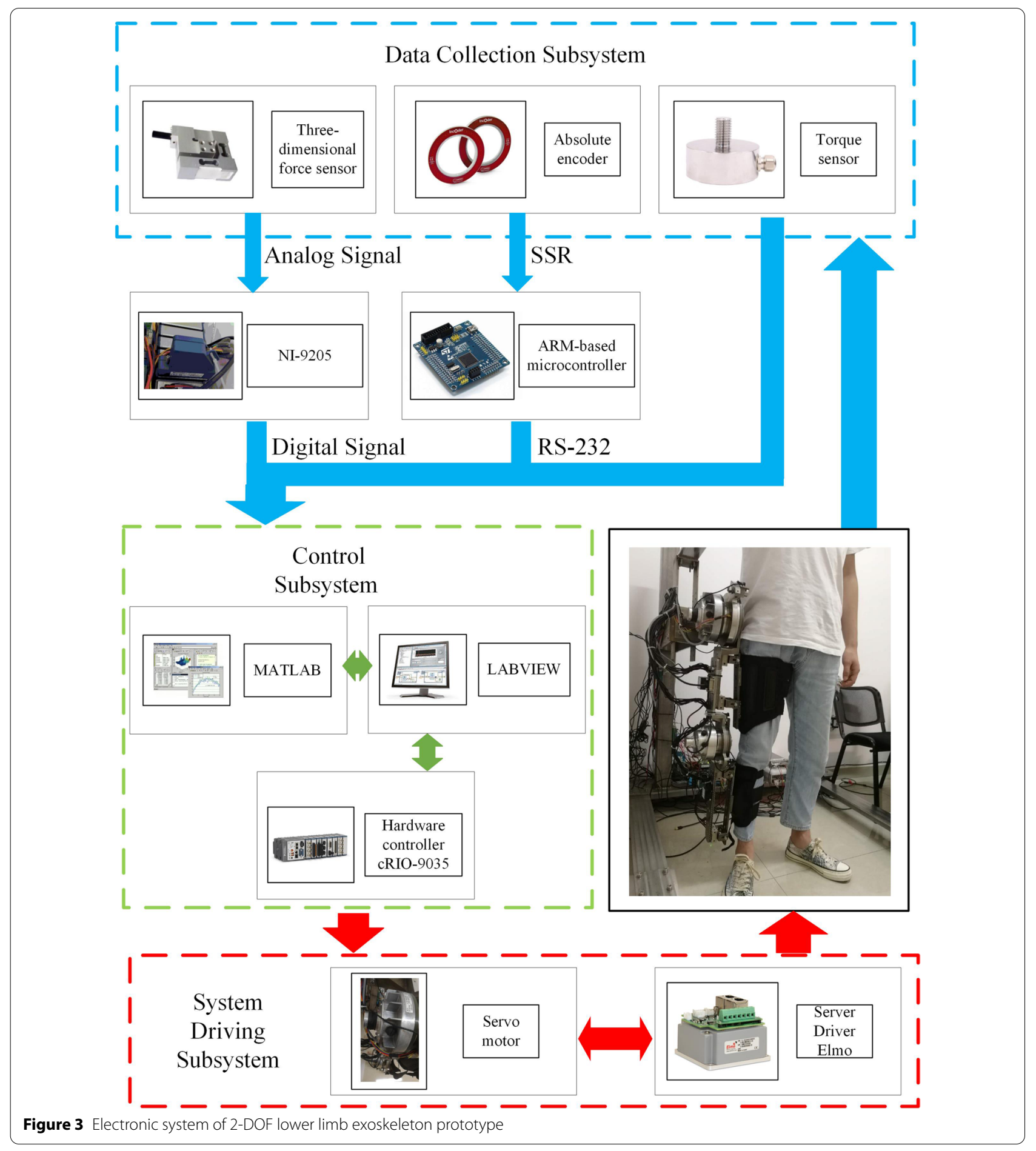




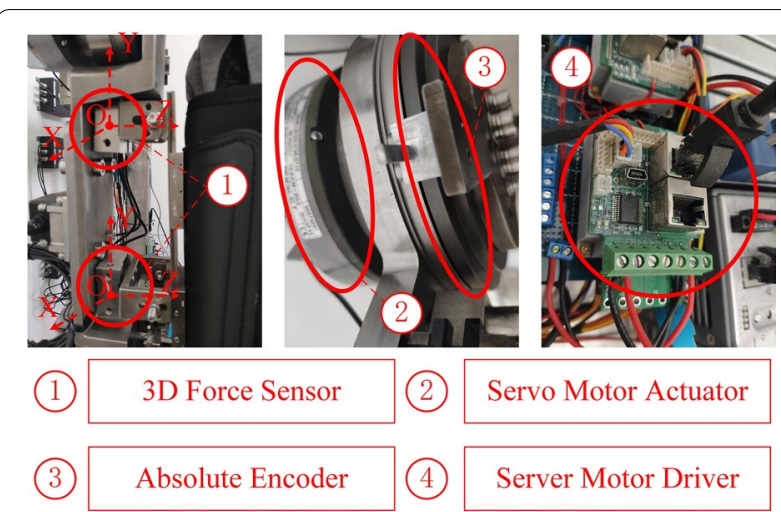

Figure 4 Sensors, actuators, and drivers are used in the exoskeleton electronic system

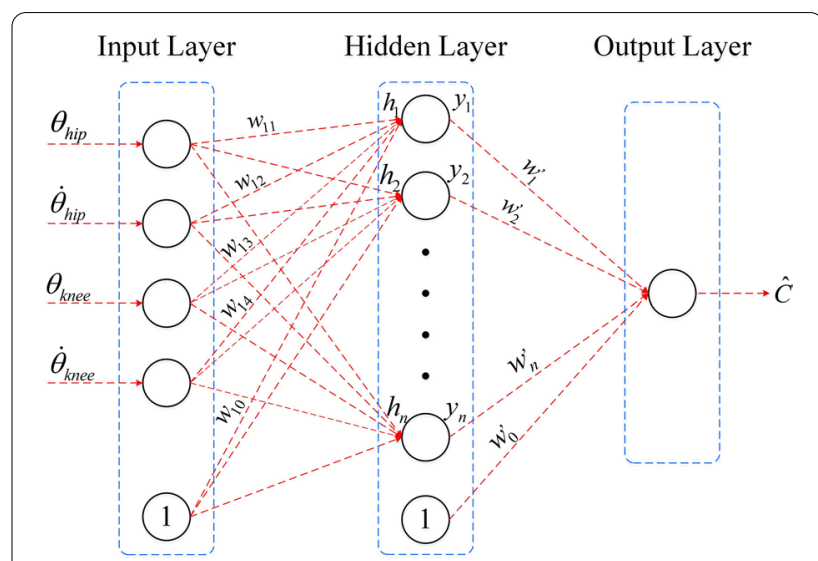

Figure 5 BP neural network structure is used for the human-robot interaction force correction

$$
y_{i}=\varphi\left(h_{i}\right)=\varphi\left(\sum_{j=0}^{4} \omega_{i j} x_{j}\right),
$$

where $x_{j}$ is the inputs to neural network, $x_{0}=1$ is the bias, $x_{1}=\theta_{\text {hip }}, x_{2}=\dot{\theta}_{\text {hip }}, x_{3}=\theta_{\text {knee }}$ and $x_{4}=\dot{\theta}_{\text {knee }}$, the function $\varphi\left(h_{i}\right)$ is the sigmoidal activation function given by

$$
\varphi\left(h_{i}\right)=\tanh \left(h_{i}\right) .
$$

The output neuron is given by

$$
\hat{C}=\sum_{i=0}^{n} \omega_{i}^{\prime} y_{i}
$$

where the neural network $\hat{C}$ is the estimated value of the force sensor error, $n$ is the number of the hidden neurons, and $y_{0}=1$.

The cost function is designed as the mean squared error (MSE)

$$
M S E=\frac{1}{M} \sum_{m=1}^{M}(C-\hat{C})^{2}
$$

where $M$ is sample points number in the training set.

\section{Control Mode of Lower Limb Exoskeleton}

According to patients' different recovery goals, the lower limb exoskeleton's working mechanism can be roughly divided into active mode and passive mode. More specifically, for the passive mode, the exoskeleton's desired trajectories are designed based on specific rehabilitation training tasks in advance. Relatively, the desired joint trajectories are generated based on the real-time human motion intention. In other words, the exoskeleton needs
Three-layer is used to compose the neural networks: an input layer, hidden layer, and output layer. For the $i$ th hidden neuron, the value is computed by 
to follow the tester's motion to realize the human-robot coordination task.

\subsection{Exoskeleton's Dynamic Model}

The dynamic of 2-DOF exoskeleton prototype is described as follows:

$$
\boldsymbol{M}(\boldsymbol{\theta}) \ddot{\boldsymbol{\theta}}+\boldsymbol{C}(\boldsymbol{\theta}, \dot{\boldsymbol{\theta}}) \dot{\boldsymbol{\theta}}+\boldsymbol{G}(\boldsymbol{\theta})+\boldsymbol{\tau}_{\mathrm{f}}(\dot{\boldsymbol{\theta}})=\boldsymbol{\tau}+\boldsymbol{\tau}_{\mathrm{dis}},
$$

where $\boldsymbol{\theta} \in \mathbb{R}^{2}$ is the position coordinates of the robotic joints, $\boldsymbol{\tau} \in \mathbb{R}^{2}$ is the input driven torque, $\boldsymbol{\tau}_{\text {dis }} \in \mathbb{R}^{2}$ is the human-robot interaction torque, $\boldsymbol{M}(\boldsymbol{\theta}) \in \mathbb{R}^{2 \times 2}$ is the inertia matrix, $\boldsymbol{C}(\boldsymbol{\theta}, \dot{\boldsymbol{\theta}}) \in \mathbb{R}^{2 \times 2}$ is the centripetal and Coriolis torques, $\boldsymbol{G}(\boldsymbol{\theta}) \in \mathbb{R}^{2}$ is the force of gravity contributed by the exoskeleton, $\boldsymbol{\tau}_{\mathrm{f}}(\dot{\boldsymbol{\theta}}) \in \mathbb{R}^{2}$ is the joint friction torque.

Lemma 1 [30]: For the terms $\boldsymbol{M}(\boldsymbol{\theta}), \boldsymbol{C}(\boldsymbol{\theta}, \dot{\boldsymbol{\theta}}), \boldsymbol{G}(\boldsymbol{\theta})$, the following properties always hold:

1) The parameter matrix $\boldsymbol{M}(\boldsymbol{\theta})$ is symmetric and positive definite.

2) The parameter matrix $\dot{\boldsymbol{M}}(\boldsymbol{\theta})-2 \boldsymbol{C}(\boldsymbol{\theta}, \dot{\boldsymbol{\theta}})$ is skew-symmetric.

\subsection{Passive Control Based on Backstepping Controller}

As shown in Figure 1, for the passive mode, to ensure the dynamic performance and system reliability, instead of the traditional PID controller, the model-based

$$
\left\{\begin{array}{l}
z_{1}=x_{1}-x_{\mathrm{r}} \\
z_{2}=x_{2}-\alpha
\end{array}\right.
$$

where the reference trajectory $x_{\mathrm{r}}$ is generated according to the human walking gait and $\alpha \in \mathbb{R}^{2}$ is the virtual control variable.

The candidate Lyapunov functions of the state space model are selected as

$$
\left\{\begin{array}{l}
V_{1}=\frac{1}{2} z_{1}^{\mathrm{T}} z_{1} \\
V_{2}=V_{1}+\frac{1}{2} z_{2}^{\mathrm{T}} \boldsymbol{M} \boldsymbol{z}_{2}
\end{array}\right.
$$

Then the derivative of $z_{1}$ yields

$$
\dot{z}_{1}=\dot{x}_{1}-\dot{x}_{\mathrm{r}}=z_{2}+\alpha-\dot{x}_{\mathrm{r}} .
$$

Hence, the derivative of $V_{1}$ is given by

$$
\dot{V}_{1}=z_{1}^{\mathrm{T}} \dot{z}_{1}=z_{1}^{\mathrm{T}}\left(z_{2}+\alpha-\dot{\boldsymbol{x}}_{\mathrm{r}}\right),
$$

and the virtual control variable $\boldsymbol{\alpha}$ is designed as

$$
\boldsymbol{\alpha}=-\boldsymbol{K}_{1} z_{1}+\dot{\boldsymbol{x}}_{\mathrm{r}}
$$

where $K_{1} \in \mathbb{R}^{2 \times 2}$ is a positive definite matrix as the first control gain.

Then $\dot{V}_{1}$ yields

$$
\dot{V}_{1}=-z_{1}^{\mathrm{T}} \boldsymbol{K}_{1} z_{1}+z_{1}^{\mathrm{T}} z_{2} .
$$

According to Lemma 1 , the time derivative of $V_{2}$ is given by

$$
\begin{aligned}
\dot{V}_{2}= & \dot{V}_{1}+\boldsymbol{z}_{2}^{\mathrm{T}} \boldsymbol{M} \dot{z}_{2}+\frac{1}{2} z_{2}^{\mathrm{T}} \dot{\boldsymbol{M}} \boldsymbol{z}_{2} \\
= & -\boldsymbol{z}_{1}^{\mathrm{T}} \boldsymbol{K}_{1} \boldsymbol{z}_{1}+\boldsymbol{z}_{1}^{\mathrm{T}} \boldsymbol{z}_{2} \\
& +\boldsymbol{z}_{2}^{\mathrm{T}}\left(\boldsymbol{\tau}+\boldsymbol{\tau}_{\mathrm{dis}}-\boldsymbol{\tau}_{\mathrm{f}}-\boldsymbol{G}-\boldsymbol{C} \boldsymbol{x}_{2}-\boldsymbol{M} \boldsymbol{\alpha}+\frac{1}{2} \dot{\boldsymbol{M}} \boldsymbol{z}_{2}\right) \\
= & -\boldsymbol{z}_{1}^{\mathrm{T}} \boldsymbol{K}_{1} \boldsymbol{z}_{1}+\boldsymbol{z}_{1}^{\mathrm{T}} \boldsymbol{z}_{2}+\boldsymbol{z}_{2}^{\mathrm{T}}\left(\boldsymbol{\tau}+\boldsymbol{\tau}_{\mathrm{dis}}-\boldsymbol{\tau}_{\mathrm{f}}-\boldsymbol{G}-\boldsymbol{C} \boldsymbol{\alpha}-\boldsymbol{M} \dot{\boldsymbol{\alpha}}\right) .
\end{aligned}
$$

controller, backstepping controller, is adopted based on the dynamic exoskeleton prototype.

For the 2-DOF exoskeleton as shown in Figure 2, according to exoskeleton dynamic (5), if the state variables are defined as $\boldsymbol{x}_{1}=\boldsymbol{\theta}=\left[\theta_{1}, \theta_{2}\right]^{\mathrm{T}}, \boldsymbol{x}_{2}=\dot{\boldsymbol{\theta}}=\left[\dot{\theta}_{1}, \dot{\theta}_{2}\right]^{\mathrm{T}}$, the state-space model is shown as follows:

$$
\left\{\begin{array}{l}
\dot{x}_{1}=\boldsymbol{x}_{2} \\
\dot{\boldsymbol{x}}_{2}=\boldsymbol{M}^{-1}\left[\boldsymbol{\tau}+\boldsymbol{\tau}_{\mathrm{dis}}-\tau_{\mathrm{f}}-\boldsymbol{G}-\boldsymbol{C} \boldsymbol{x}_{2}\right] .
\end{array}\right.
$$

The state error of exoskeleton $\boldsymbol{z}_{1}, \boldsymbol{z}_{2} \in \mathbb{R}^{2}$ are defined as follows:
Finally, the backstepping controller is designed as follows:

$$
\boldsymbol{\tau}=-\boldsymbol{z}_{1}-\boldsymbol{K}_{2} z_{2}-\boldsymbol{\tau}_{\mathrm{dis}}+\boldsymbol{G}+\boldsymbol{C} \boldsymbol{\alpha}+\boldsymbol{M} \dot{\boldsymbol{\alpha}}+\boldsymbol{\tau}_{\mathrm{f}}
$$

where $K_{2} \in \mathbb{R}^{2 \times 2}$ is a positive definite matrix as the second control gain.

Substituting Eq. (14) into Eq. (13), we have

$$
\begin{gathered}
\dot{V}_{2}=-\boldsymbol{z}_{1}^{\mathrm{T}} \boldsymbol{K}_{1} \boldsymbol{z}_{1}+\boldsymbol{z}_{1}^{\mathrm{T}} \boldsymbol{z}_{2}+\boldsymbol{z}_{2}^{\mathrm{T}}\left(\boldsymbol{\tau}+\boldsymbol{\tau}_{\mathrm{dis}}-\boldsymbol{\tau}_{\mathrm{f}}-\boldsymbol{G}-\boldsymbol{C} \boldsymbol{\alpha}-\boldsymbol{M} \dot{\boldsymbol{\alpha}}\right) \\
=-\boldsymbol{z}_{1}^{\mathrm{T}} \boldsymbol{K}_{1} \boldsymbol{z}_{1}-\boldsymbol{z}_{2}^{\mathrm{T}} \boldsymbol{K}_{2} \boldsymbol{z}_{2} \leq 0 .
\end{gathered}
$$

Hence, the system state errors $z_{1}$ and $z_{2}$ converge to zero as $t \rightarrow \infty$. 


\subsection{Active Control Based on Admittance Controller}

For the exoskeleton active mode, as shown in Figure 1, to achieve the active control objective, a type of force/ position dual-control scheme is adopted based on realtime human-robot interaction. More specifically, the reference trajectory is generated in real-time by the admittance controller for the outer force loop. Then, the inner position loop is designed by the backstepping controller (14).

The admittance control is adopted to correspond to the direct intention to motivate and achieve compliant control. And based on the mechanical admittance concept, the target dynamic behavior between the motion of the exoskeleton's joint/end-effector and the human-robot interaction (torque/force) is realized. In this paper, the admittance concept is built on a joint coordinate. Therefore, the target admittance $\boldsymbol{\theta} \in \mathbb{R}^{2}$ and $\boldsymbol{\tau}_{\text {dis }} \in \mathbb{R}^{2}$ can be usually expressed as a second-order system:

$$
\boldsymbol{M}_{\mathrm{im}}\left(\ddot{\boldsymbol{\theta}}-\ddot{\boldsymbol{\theta}}_{\mathrm{c}}\right)+\boldsymbol{B}_{\mathrm{im}}\left(\dot{\boldsymbol{\theta}}-\dot{\boldsymbol{\theta}}_{\mathrm{c}}\right)+\boldsymbol{K}_{\mathrm{im}}\left(\boldsymbol{\theta}-\boldsymbol{\theta}_{\mathrm{c}}\right)=\boldsymbol{\tau}_{\mathrm{dis}},
$$

where $\boldsymbol{\theta}_{\mathrm{c}} \in \mathbb{R}^{2}$ denotes the equilibrium position of exoskeleton joint, $\boldsymbol{M}_{\mathrm{im}}, \boldsymbol{B}_{\mathrm{im}}, \boldsymbol{\theta}, \dot{\boldsymbol{\theta}} \in \mathbb{R}^{2}$ are the virtual inertia, virtual damp, and virtual stiffness respectively, which usually are designed in the form of positive definite diagonal matrices generally, $\boldsymbol{\theta}, \dot{\boldsymbol{\theta}} \in \mathbb{R}^{2}$ are the joint position and joint angular velocity respectively. As shown in Figure 1, using the sampling human-robot interaction torque $\boldsymbol{\tau}_{\text {dis }}, \boldsymbol{\theta}$ can be calculated and set as the reference trajectory $\boldsymbol{\theta}_{\mathrm{r}}$ of position control loop. Accordingly, as long as the inner loop position controller's performance is outstanding enough, the designed dynamic (16) can be established.

For the exoskeleton cooperative walking experiment, the virtual inertia $\boldsymbol{M}_{\mathrm{im}}$ is set as $\boldsymbol{0}$. Furthermore, since the motion gaits of the different operators have otherness widely, it is arduous to predict the operator's motion intention in advance. Consequently, the equilibrium position $\boldsymbol{\theta}_{\mathrm{c}}$ can be set as the current joint position. Generally speaking, without human-robot interaction, the exoskeleton is considered to have completed the cooperative walking task perfectly at the current moment, and the exoskeleton robot should remain at the current position. Consequently, the discrete form of admittance controller is rewritten at $t+\Delta$ as follows:

$$
\boldsymbol{B}_{\mathrm{im}}\left[\dot{\boldsymbol{\theta}}_{\mathrm{r}}(t+\Delta)-\dot{\boldsymbol{\theta}}(t)\right]+\boldsymbol{K}_{\mathrm{im}}\left[\boldsymbol{\theta}_{\mathrm{r}}(t+\Delta)-\boldsymbol{\theta}(t)\right]=\boldsymbol{\tau}_{\mathrm{dis}}(t+\Delta),
$$

where $\Delta$ denotes the control interval of exoskeleton prototype, $\boldsymbol{\theta}(t)$ denotes the joint position at time $t, \dot{\boldsymbol{\theta}}_{\mathrm{r}}(t+\Delta)$,
$\boldsymbol{\theta}_{\mathrm{r}}(t+\Delta) \in \mathbb{R}^{2 \times 2}$ denote the reference joint angular velocity and joint position respectively at time $t+\Delta$, and $\boldsymbol{\theta}_{\mathrm{r}}(t+\Delta)$ is obtained based on $\boldsymbol{\tau}_{\text {dis }}(t+\Delta)$ and designed admittance parameters $\boldsymbol{B}_{\mathrm{im}}$ and $\boldsymbol{K}_{\mathrm{im}}$.

\section{Variable Admittance Parameter Strategy}

For the admittance controller with fixed admittance parameters, the response time and the control accuracy exhibit an inverse relationship. And for the complex application scenarios, fixed admittance control is difficult to express excellent performance and meet specific requirements. More specifically, if the operator walks with higher step frequency, the smaller admittance parameters need to be set to reduce human-robot impedance, which guarantees the faster dynamic response and the exoskeleton's favor flexibility. On the contrary, for the operator with lower step frequency, the dynamic response speed requirement in the human-robot coupling system is reduced. Hence, the higher admittance parameters are set and increase the human-robot impedance, which improves the steady control accuracy and system stability.

The variable admittance parameter strategy needs to be formulated to guarantee the operator's wearable comfort and safety in different applications, reflected by the real-time human-robot interaction torque. Since the exoskeleton flexibility is contradicted with its stability, the admittance parameter regulation is essential to design the variable admittance controller in different conditions.

According to the above analysis and discussion, the admittance parameters can be regulated for operators with similar height and weight based on the real-time step frequency to guarantee the wearable comfortable effect of the human-robot coupling system. Notably, a type of wearable comfortable evaluate factor needs to be defined to balance the flexibility and control accuracy for different step frequencies. In this paper, the statistical experiment is realized to evaluate wearable comfortable and formulate a variable admittance strategy.

In the statistical experiment, based on the fixed admittance controller, the operator (age: 25 years old, height: $180 \mathrm{~cm}$, and weight: $57.4 \mathrm{~kg}$ ) completed multiple sets of exoskeleton cooperative walking experiments with different step frequency and different admittance parameters. Simultaneously, several real-time data have been recorded by Labview, such as human-robot interaction torque, two joint angle. The exoskeleton cooperative walking experiments had respective $K=5000$ sampling points with seven groups according to the step frequency (24 step/ $\mathrm{min}, 26 \mathrm{step} / \mathrm{min}, 28 \mathrm{step} / \mathrm{min}, 30 \mathrm{step} / \mathrm{min}, 32 \mathrm{step} / \mathrm{min}$, 
$34 \mathrm{step} / \mathrm{min}$, and $36 \mathrm{step} / \mathrm{min})$. And the data sampling period is $T=10 \mathrm{~ms}$. Moreover, the virtual damping and virtual stiffness of two joints are regulated to keep their ratio as follows:

$$
\begin{aligned}
& \boldsymbol{B}_{\mathrm{im}}=\left[\begin{array}{cc}
B_{\text {hip }} & 0 \\
0 & B_{\text {knee }}
\end{array}\right]=\beta\left[\begin{array}{cc}
B_{\text {hip }, 0} & 0 \\
0 & B_{\text {knee }, 0}
\end{array}\right], \\
& \boldsymbol{K}_{\mathrm{im}}=\left[\begin{array}{cc}
K_{\text {hip }} & 0 \\
0 & K_{\text {knee }}
\end{array}\right]=\beta\left[\begin{array}{cc}
K_{\text {hip }, 0} & 0 \\
0 & K_{\text {knee }, 0}
\end{array}\right],
\end{aligned}
$$

where $\beta$ denotes the scale factor, $B_{h i p, 0}=0.045, K_{h i p, 0}=$ $0.04, B_{\text {knee }, 0}=0.022$ and $K_{\text {knee }, 0}=0.037$ denote the basic admittances of two joint coordinates. Hence, the admittance parameters are unified regulated by adjusting $\beta$.

\subsection{Stability Evaluation with Different Step Frequency}

By referring to the definition of the instability index proposed in Refs. [21, 22], the corresponding stability index in the angle coordinate system for the exoskeleton is defined as follows:

$$
\begin{aligned}
& I_{\omega}[K T]=\left(\frac{\sum_{\omega=\omega_{c}}^{\omega_{s} / 2} P_{\tau, \text { hip }}(\omega)}{2 \sum_{\omega=0}^{\omega_{s} / 2} P_{\tau, \text { hip }}(\omega)}-P_{0, \text { hip }}\right)^{2} \\
&+\left(\frac{\sum_{\omega=\omega_{c}}^{\omega_{s} / 2} P_{\tau, \text { knee }}(\omega)}{2 \sum_{\omega=0}^{\omega_{s} / 2} P_{\tau, \text { knee }}(\omega)}-P_{0, \text { knee }}\right. \\
&)^{2}
\end{aligned}
$$

where $T$ is the sampling interval, $\omega_{0}$ is the lowest frequency of the Fast Fourier Transform (FFT), $\omega_{s} / 2$ is determined by the Nyquist sampling theorem, $P_{\tau, h i p}(\omega)$ and $P_{\tau, k n e e}(\omega)$ are the magnitudes of the $\omega_{c}$ frequency component derived from the FFT of two interaction torque signals, $P_{0, \text { hip }}(\omega)$ and $P_{0, \text { knee }}(\omega)$ are the two joint thresholds to address the sampling noise and the other irrelevant high frequency signal. The crossover frequency $\omega_{c}=2 \mathrm{~Hz}$ is determined by experiment for two joints. Two magnitudes $P_{0, \text { hip }}(\omega)$ and $P_{0, \text { knee }}(\omega)$ are selected as 0.2883 and 0.3120 for two joints, respectively.

The stability index $I_{\omega}[K T]$ is defined as the sum of squares for the ratio of the high-frequency components $P_{\tau, \text { hip }}(\omega)$ and $P_{\tau, \text { knee }}(\omega)$ to all the frequencies components. Meanwhile, it is also considered to be the instability evaluation of the admittance controller. By analyzing the statistical experimental results, as shown in Figure 6, with the particular operator's step frequency, as the scale factor $\beta$ increases, the corresponding stability factor generally shows a decreasing trend. Hence, compared with the lower scale factor $\beta$, the exoskeleton humanrobot coupling system with a higher scale factor $\beta$ shows more excellent stability.

\subsection{Human-robot Interaction Index with Different Step Frequency}

For the human-robot cooperative motion, the tolerable interaction torque is one important objective to evaluate the suitable exoskeleton. Hence, it is necessary to design a reasonable scale factor to guarantee a small interaction torque. The human-robot interaction index for the exoskeleton is defined as follows:

$$
I_{\tau, \text { ext }}[K T]=\left(\frac{1}{K} \sum_{i=0}^{K}\left|\tau_{\text {ext,hip,i }}\right|\right)^{2}+\left(\frac{1}{K} \sum_{i=0}^{K}\left|\tau_{\text {ext,knee }, i}\right|\right)^{2},
$$

where $\tau_{\text {ext, hip }, i}$ and $\tau_{\text {ext,knee, } i}$ are two joint interaction torques of the $i$ th sampling data. The human-robot interaction index $I_{\tau, \text { ext }}[K T]$ is the sum of squares for two joint interaction torques.

By analyzing the statistical experimental results, as shown in Figure 7, with the particular operator's step frequency, as the scale factor $\beta$ increases, the human-robot interaction index generally shows an increasing trend. Hence, compared with the higher scale factor $\beta$, the exoskeleton human-robot coupling system with the lower scale factor $\beta$ shows more excellent flexibility and operator's freedom.

\subsection{Wearable Comfortable Performance with Different Step Frequency}

Both frequency oscillations and the magnitude of humanrobot interaction torque are the essential factors affecting wearable comfortable. Considering the above statistical experimental results, it is reasonable to define the wearable, comfortable performance to evaluate wearable comfortable by balancing the two elements mentioned above. Based on the stability index (20) and human-robot interaction index (21), the wearable comfortable performance of 2-DOF lower limb exoskeleton is defined to overall evaluate the favorable admittance controller as follows:

$$
I[K T]=K_{\text {Fre }} I_{\omega}[K T]+K_{\text {Tor }} I_{\tau, \text { ext }}[K T],
$$

where $I_{\omega}[K T]$ and $I_{\tau, \text { ext }}[K T]$ denote the gain of the stability index and human-robot interaction index respectively. In this study, $K_{\text {Fre }}$ and $K_{\text {Tor }}$ are selected as 900 and 1 , respectively. The statistical experiment for the wearable comfortable performance is shown in Figure 8. For the different step frequency (24 step/minute, $26 \mathrm{step} / \mathrm{min}, 28$ step $/ \mathrm{min}, 30 \mathrm{step} / \mathrm{min}, 32 \mathrm{step} / \mathrm{min}, 34 \mathrm{step} / \mathrm{min}$, and 36 


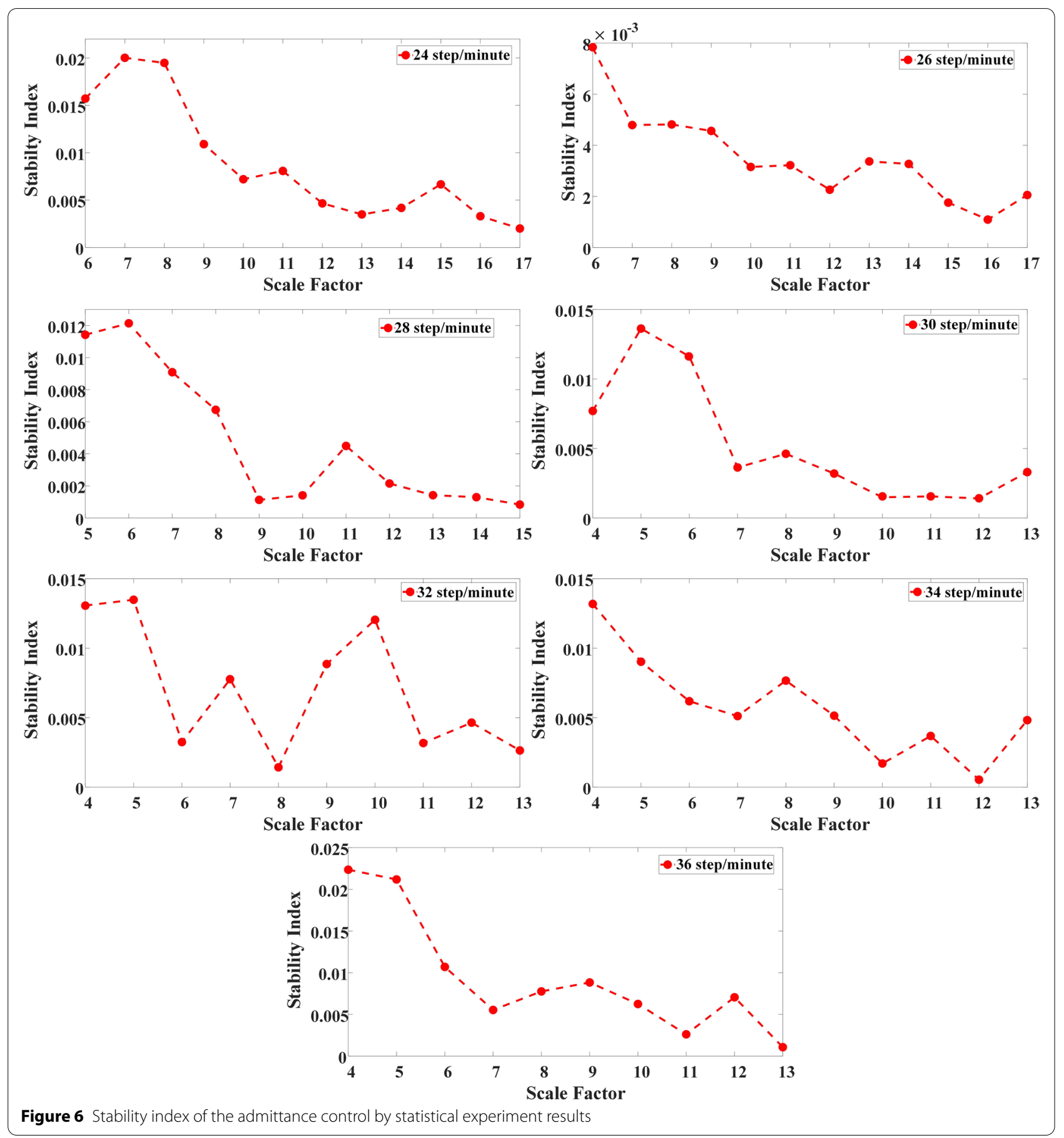

step $/ \mathrm{min}$ ), the corresponding optimal scale factor $\beta$ is 13 , $13,10,8,8,6$, and 6 , respectively.

Hence, based on the corresponding optimal scale factor in different step frequencies, the variable admittance strategy is developed by function fitting for the step frequency range from $24 \mathrm{step} / \mathrm{min}$ to $36 \mathrm{step} / \mathrm{min}$ as follows:

$$
\beta=P_{1} \omega^{4}+P_{2} \omega^{3}+P_{3} \omega^{2}+P_{4} \omega+P_{5},
$$

where $\omega$ denotes the real-time step frequency, $P_{1}=$ $-0.002367, P_{2}=0.291, P_{3}=-13.29, P_{4}=266.5$ and $P_{5}=$ -1965 denote the fitting coefficient. 

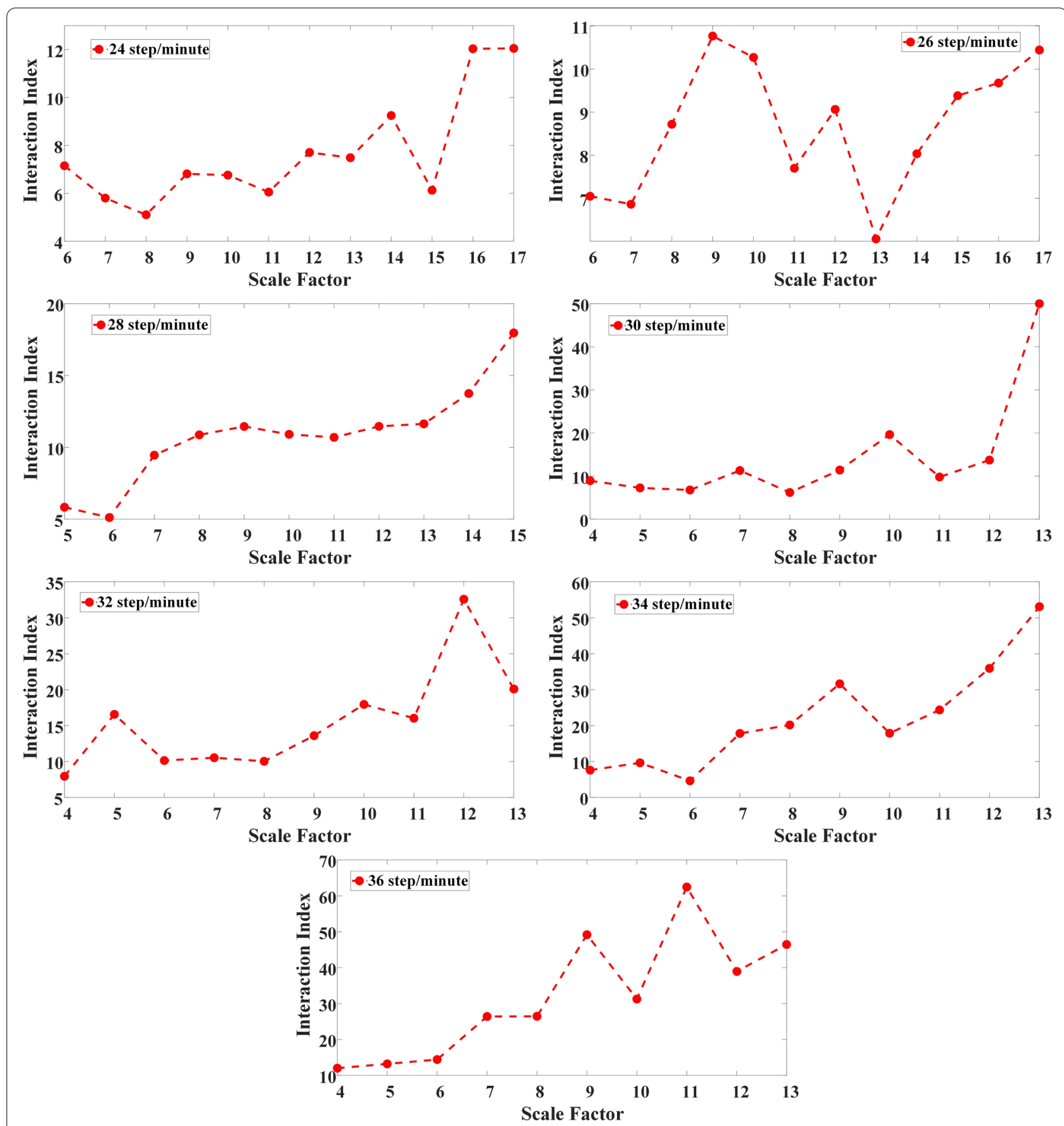

Figure 7 Human-robot interaction index of the admittance control by statistical experiment results

\section{Experiment}

\subsection{Stability Evaluation with Different Step Frequency}

For the error correction experiment of the 3-D force sensor, the 12 BP neural networks (Thigh-Down-X, Thigh-Down-Y, Thigh-Down-Z, Thigh-Up-X, ThighUp-Y, Thigh-Up-Z, Shank-Down-X, Shank-Down-Y,
Shank-Down-Z, Shank-Up-X, Shank-Up-Y, Shank-Up-Z) with the structure as shown in Figure 5 had been trained by the sampling set. Furthermore, the neuron number is 60 , the network learning rate is 0.1 , and the setting training epoch is 1000 . The data for both train and verification sample set include two joint angles, angular velocities, 

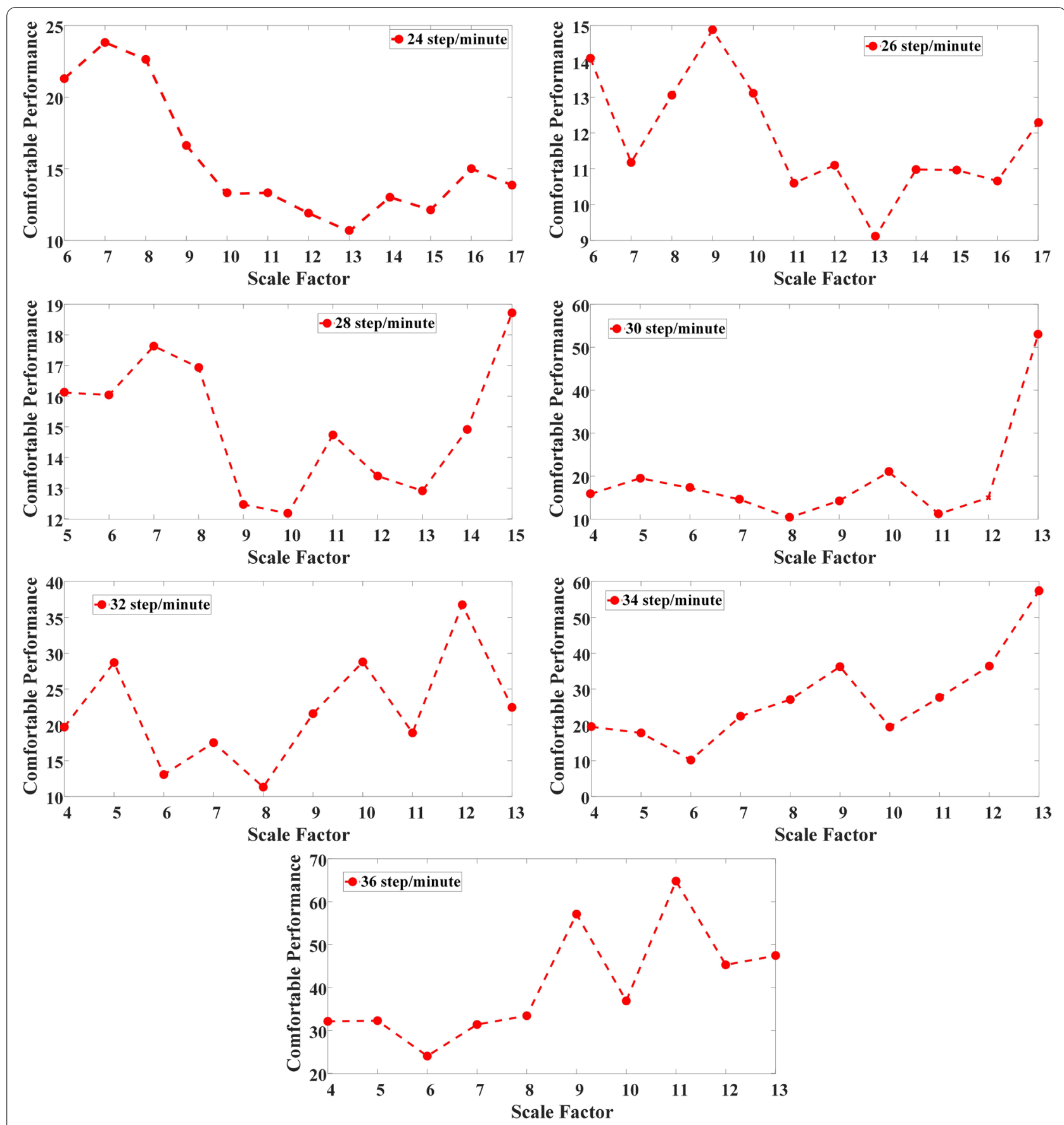

Figure 8 Wearable comfortable performance by using the admittance controller

and sensor forces. Meanwhile, the data had been randomly divided into two types of samples in a fixed proportion. In this study, the setting train sample number is 5000 , and the verification sample number is 1000 . Considering the training process of the BP neural network, the neural network performance on the train set, validation set, and test set are shown in Figure 9.
As shown in Figure 10, the performance on the validation sample set by the BP neural networks indicates that the average prediction error percent of Thigh-Down-X, Thigh-Down-Y, Thigh-Down-Z, Thigh-Up-X, Thigh-UpY, Thigh-Up-Z, Shank-Down-X, Shank-Down-Y, ShankDown-Z, Shank-Up-X, Shank-Up-Y, Shank-Up-Z are 3.3728\%, 5.3527\%, 1.9347\%, 1.4701\%, 1.0529\%, 13.3203\%, 

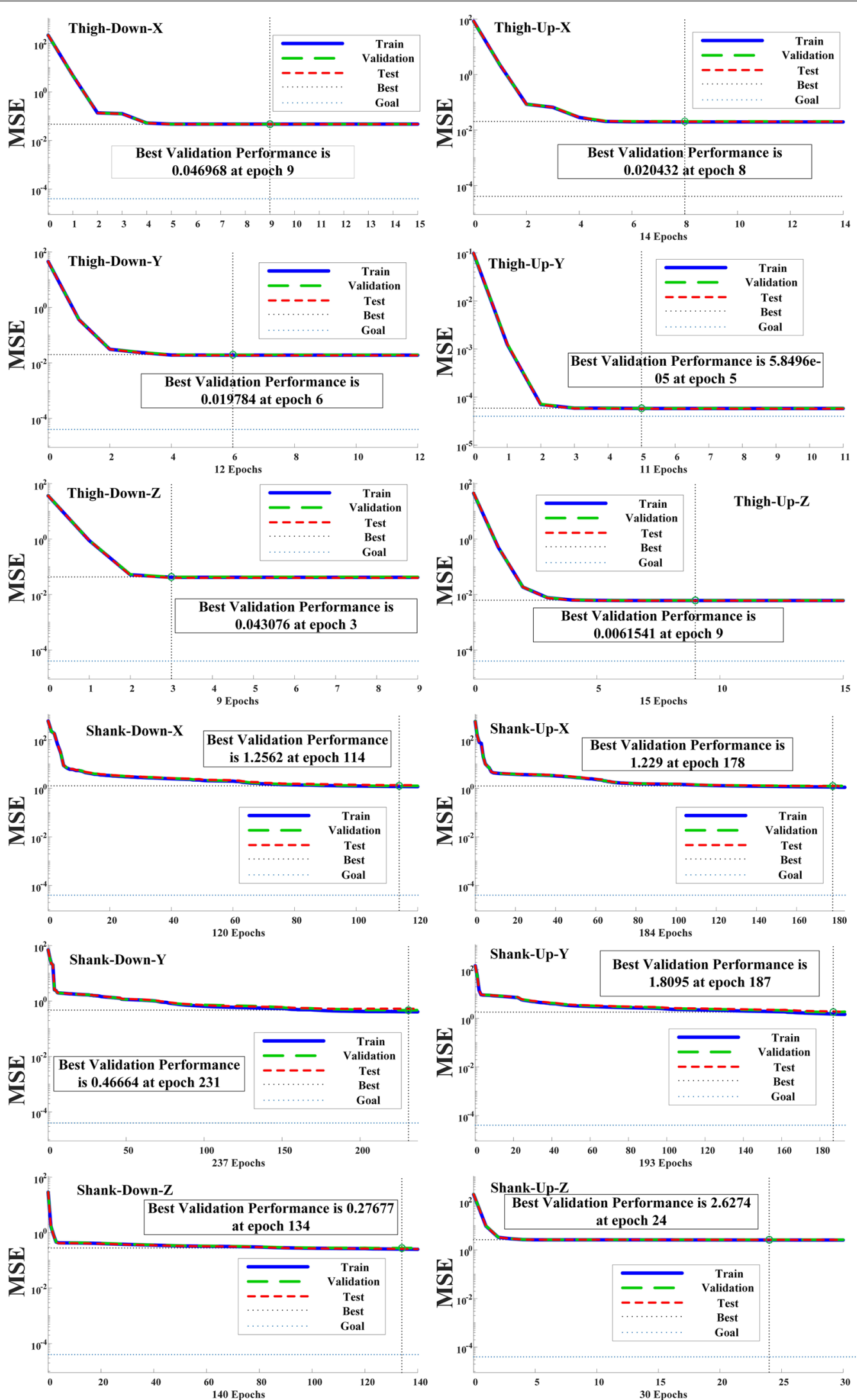

Figure 9 Training process of BP neural network 

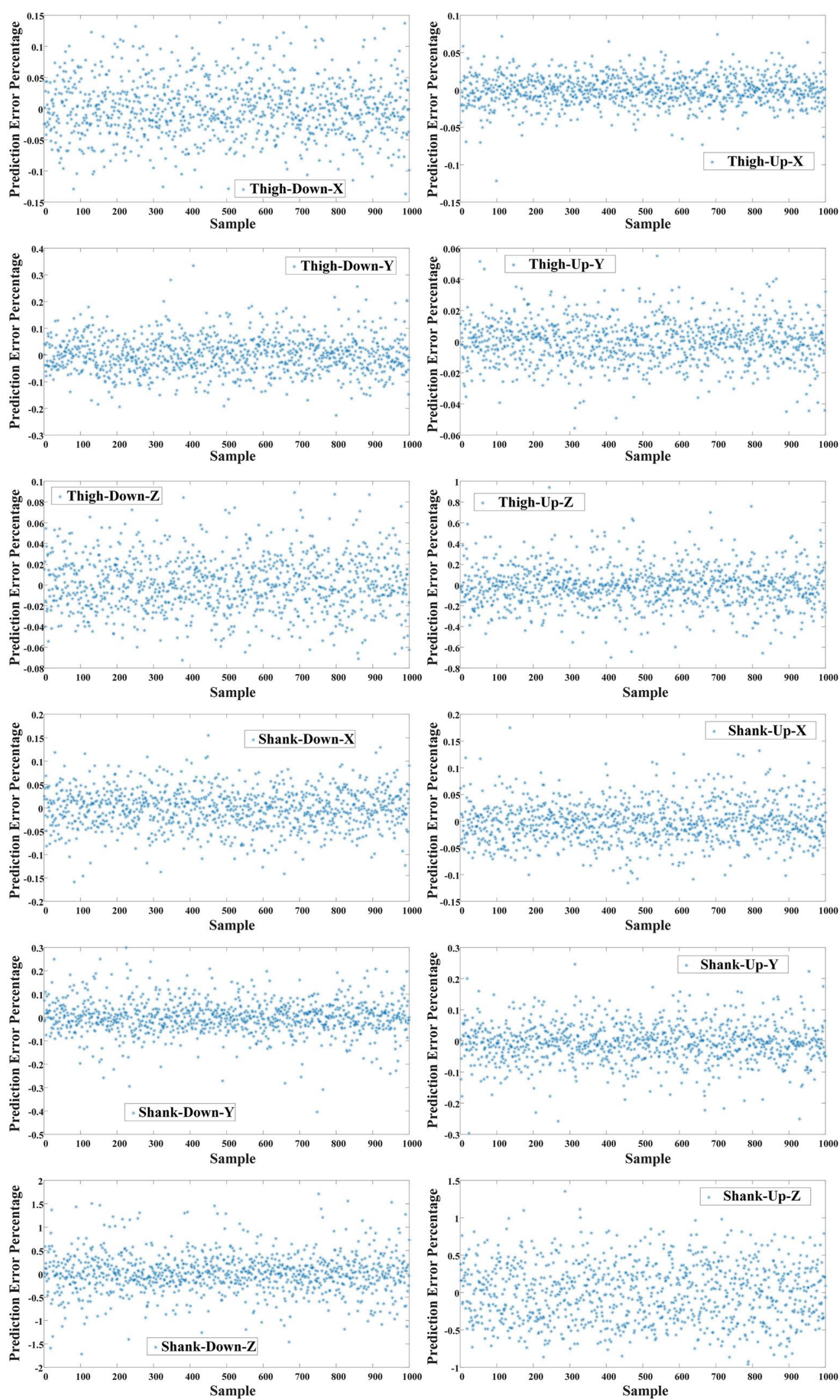

Figure 10 Prediction error percentage of the 3-D force/torque sensor by using 12 BP neural networks 
$3.1732 \%, \quad 5.4756 \%, \quad 30.3940 \%, \quad 2.8546 \%, \quad 4.5779 \%$, $31.6092 \%$, respectively. Since the Z-dimension sensor force is mainly caused by unknown external disturbance, the prediction error percentage is higher, and the prediction accuracy is relatively low. Hence, the error correction experiment of the 3-D force sensor is satisfactory.

\subsection{Backstepping Controller in Exoskeleton Passive Mode}

The human walking gaits $\theta_{1 d}$ and $\theta_{2 d}$ for the desirable trajectory are given by

$$
\begin{aligned}
& \theta_{1 d}=\sum_{k=1}^{4}\left(a_{k, \text { hip }} \sin (k \omega t)+b_{k, \text { hip }} \cos (k \omega t)\right)+\theta_{0, \text { hip }}, \\
& \theta_{2 d}=\sum_{k=1}^{4}\left(a_{k, \text { knee }} \sin (k \omega t)+b_{k, \text { knee }} \cos (k \omega t)\right)+\theta_{0, \text { knee }},
\end{aligned}
$$

where $\omega=0.4 \pi, a_{k, h i p}(k=1,2,3,4)$ are $-2.874,-2.423$, 1.227 and $-0.1462, \quad b_{k, h i p}(k=1,2,3,4)$ are 18.53, -2.016 , and -0.3704 and $0.201, \theta_{0, h i p}=10.07, a_{k, \text { knee }}$ $(k=1,2,3,4)$ are $17.62,-2.469,-3.82$ and -0.1346 , $b_{k, \text { knee }}(k=1,2,3,4)$ are $-1.494,11.72,1.014$ and 0.2165 , and $\theta_{0, k n e e}=-17.49$.

For the backstepping controller (14), the parameters $\boldsymbol{M}=\left[25.68+2.65 \cos \theta_{2}, \quad 6.91+1.32 \cos \theta_{2}\right.$; $\left.6.91+1.32 \cos \theta_{2}, 6.91\right], \boldsymbol{C}=\left[-1.32 \sin \theta_{2} \dot{\theta}_{2},-1.32 \sin \theta_{2}\right.$ $\left.\left(\dot{\theta}_{1}+\dot{\theta}_{2}\right) ; \quad 1.32 \sin \theta_{2} \dot{\theta}_{1}, \quad 0\right], \quad \boldsymbol{G}=\left[33.32 \sin \left(\theta_{1}+\theta_{2}\right)+\right.$ $\left.78.39 \sin \theta_{1} ; 33.32 \sin \left(\theta_{1}+\theta_{2}\right)\right]$, and $\boldsymbol{\tau}_{\mathbf{f}}=\left[31.38 \operatorname{sgn}\left(\dot{\theta}_{1}\right)+\right.$

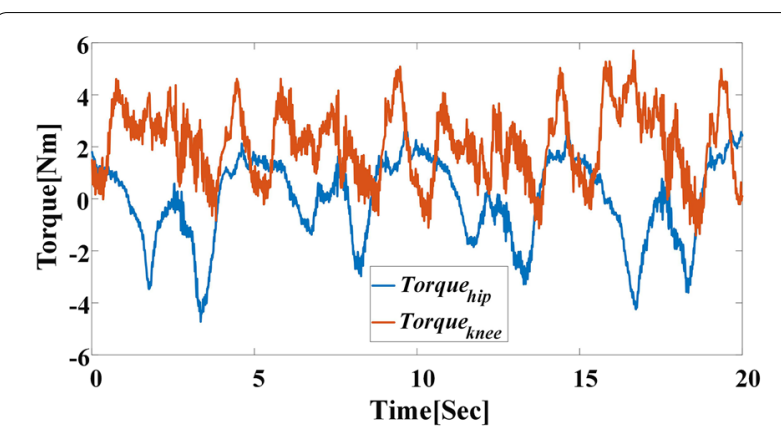

Figure 12 Human-robot interaction torque by using the backstepping controller in exoskeleton passive mode

$\left.15.55 \dot{\theta}_{1} ; \quad 43.67 \operatorname{sgn}\left(\dot{\theta}_{2}\right)+27.51 \dot{\theta}_{1}\right]$ are adopted in experiment. The control parameters are set as $\boldsymbol{K}_{1}=\operatorname{diag}\{15,15\}$ and $\boldsymbol{K}_{2}=\operatorname{diag}\{150,150\}$.

The angle responses of two exoskeleton joints and the corresponding tracking errors are shown in Figure 10, which indicates the satisfactory tracking performance $\left(\left|e_{1}\right|<0.016 \mathrm{rad},\left|e_{2}\right|<0.04 \mathrm{rad}\right)$. Furthermore, the human-robot interaction torques of the two joints are shown in Figure 11. Both interaction torques Torque $_{\text {hip }}$ and Torque knee $_{\text {are less than }} 6 \mathrm{~N} \cdot \mathrm{m}$ by using the backstepping controller. These interaction torques are acceptable for the experienced tester. Therefore, the tester can follow the walking gait of the exoskeleton with minor tolerable impedance (Figure 12).

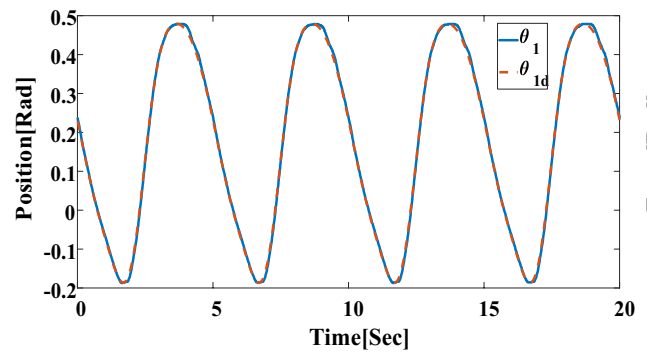

a hip angle position

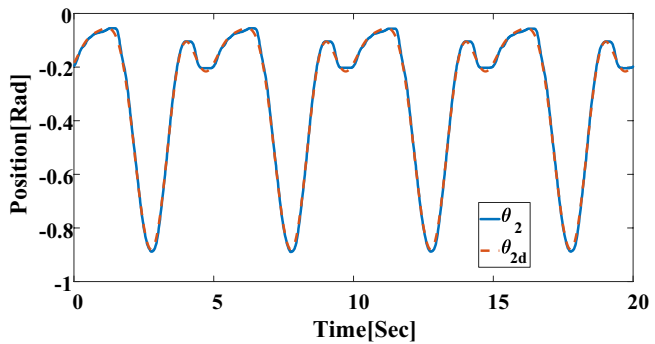

c knee angle position

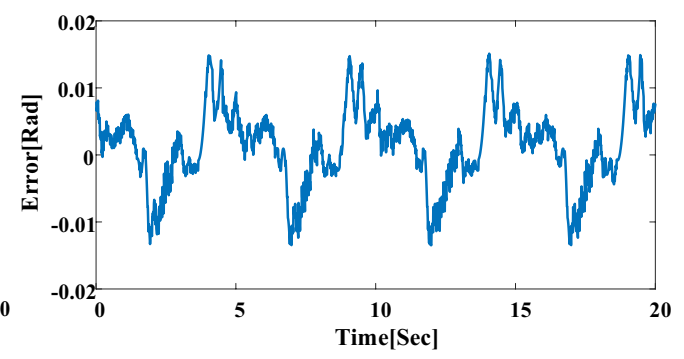

b hip angle position error

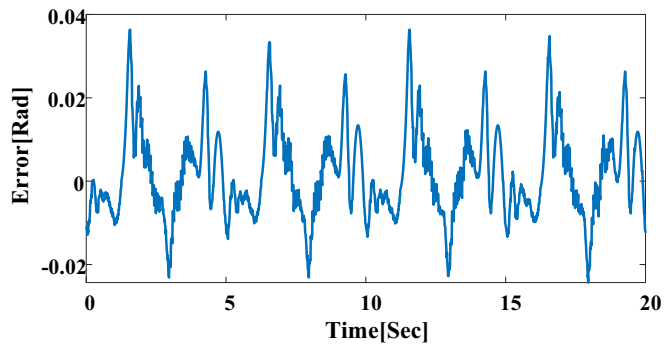

d knee angle position error

Figure 11 Two joint angle positions and errors by using the backstepping controller in exoskeleton passive mode 


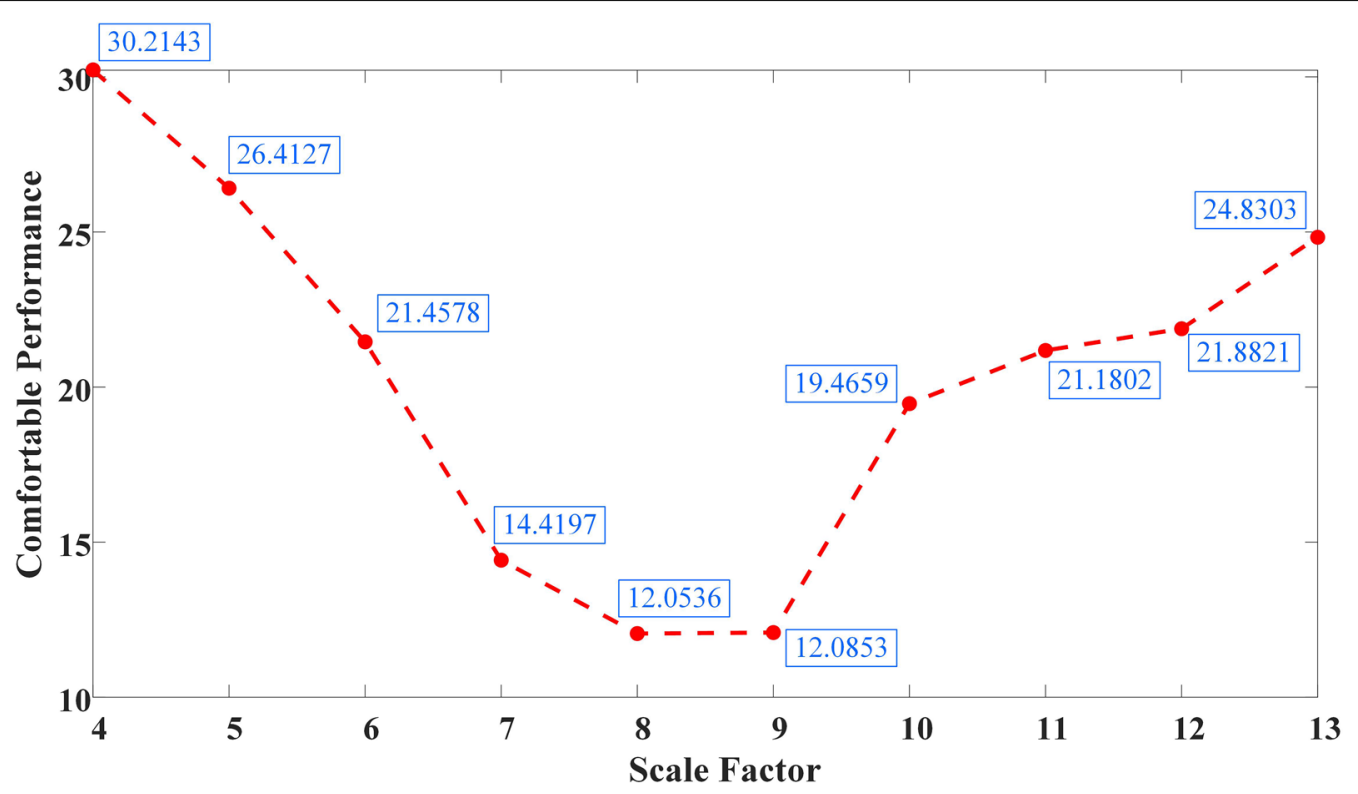

Figure 13 The wearable comfortable performance by using the constant admittance controller in different step frequency experiments

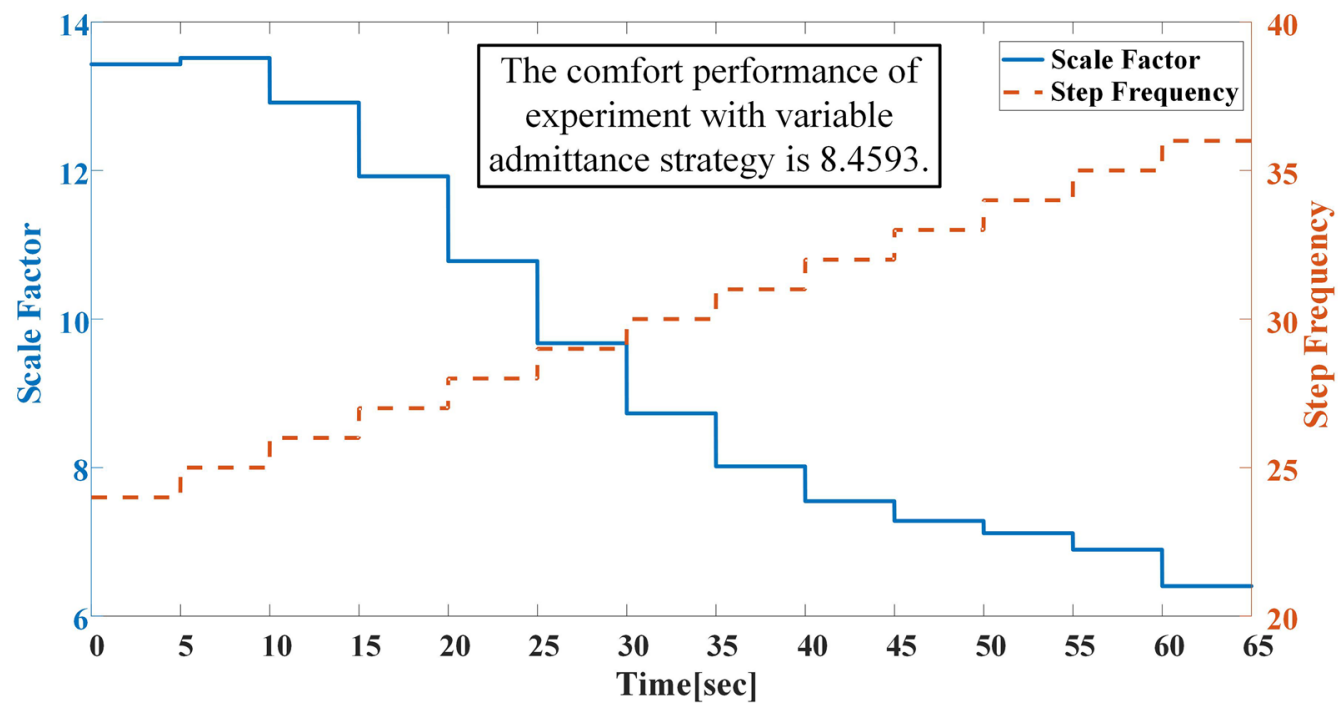

Figure 14 Variable scale factor $\beta$ (23) and the transient walking frequency using the variable admittance controller in $65 \mathrm{~s}$ time

\subsection{Variable Admittance Controller in Exoskeleton Active Mode}

To verify the admittance controller's effectiveness with a variable admittance strategy (23), the comparative experiments had been conducted based on the conception of wearable comfortable performance (22). Considering the people's daily walking step frequency is time-varying, the step frequency varying rule of all 11 comparative experiments has set as increasing from $24 \mathrm{step} / \mathrm{min}$ to $36 \mathrm{step} /$ min in $65 \mathrm{~s}$ evenly. More specifically, the 10 comparative experiments adopted traditional fixed admittance controller with different scale factors $\beta$ from 4 to 13 , and another set of experiments used variable admittance controller. The wearable comfortable performance (22) of 10 comparative experiments is shown in Figure 13. Obviously, by analyzing the statistical experimental results, the best wearable comfortable performance is 12.0536 when $\beta$ is set as 8 , and $\beta$ that is too big or too small may express the worse wearable comfortable performance 


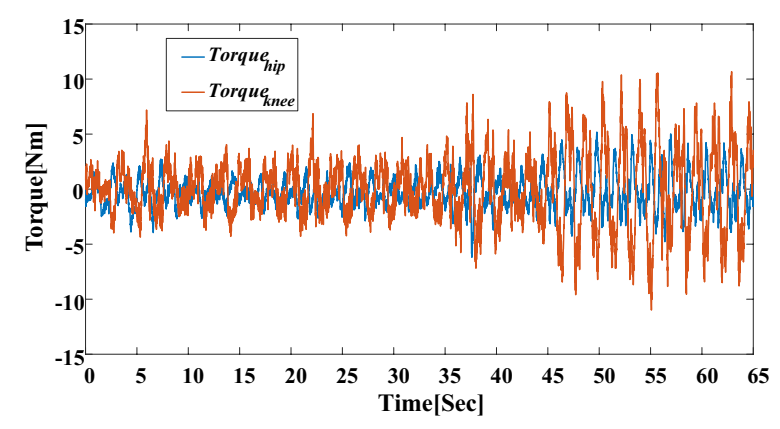

Figure 15 Human-robot interaction torque by using the variable admittance controller in exoskeleton active mode

because of high-frequency oscillations or bigger magnitude of human-robot interaction.

Meanwhile, for the variable admittance experiment, the varying trend of step frequency and corresponding variable scale factor $\beta$, which is increased from 6.4 to 13.4, based on variable admittance strategy (23) shown in Figure 14. By analyzing the statistical experimental result, the wearable comfortable performance is 8.4593. Hence, compared with the fixed admittance controller, the variable admittance controller has demonstrated more beneficial wearable comfortable performance in the human-robot cooperative walk with variable step frequency. Theoretically, the variable admittance strategy (23) adjusts the $\beta$ in real-time according to the operator's step frequency to effectively meet and balance the stability and flexibility requirements of the human-robot coupling system. However, it is difficult for fixed admittance controller to take care of different walking states.

Eventually, two human-robot interaction torques by using the variable admittance controller in exoskeleton active mode are shown in Figure 15, and the corresponding human-robot interaction torques Torque ${ }_{\text {hip }}$ and Torque $_{\text {knee }}$ are less $7 \mathrm{~N} \cdot \mathrm{m}$ and $13 \mathrm{~N} \cdot \mathrm{m}$ respectively, which implies that the human-robot impedance of the collaborative motion in transient walking frequency is constrained in a certain boundary.

\section{Conclusions}

In this study, the 2-DOF lower limb exoskeleton prototype is introduced. Firstly, the BP neural network is used to estimate and correct the 3-D force/torque sensor's real-time measurement error. Then the backstepping controller (14) is designed to realize the exoskeleton's passive control mode, which means that the person follows the exoskeleton with tolerable impedance. Subsequently, a variable admittance controller is adopted to implement the exoskeleton's active control mode and improves the human-robot wearable comfortable performance. The admittance parameters are designed according to both the human-robot interaction index and the stability index in different step frequencies of the human-robot cooperative walk. Finally, the proposed control schemes are verified by the exoskeleton experimental prototype. They indicate that the variable admittance controller has favorable wearable comfortable performances for a human-robot cooperative walk in different step frequencies in the exoskeleton active mode. Furthermore, the backstepping controller (14) guarantees the desired trajectory's good position tracking performance with tolerable human-robot interaction torque.

\section{Acknowledgements}

Not applicable.

\section{Authors' Contributions}

ZC is for writing manuscript, implement, Data verication; QG is for conceptualization, methodology; $\mathrm{HX}$ is for software programming; $\mathrm{DJ}$ is for result checking; $Y Y$ is for validation. All authors read and approved the final manuscript.

\section{Authors' Information}

Zhenlei Chen, born in 1992, is currently a PhD candidate at School of Aeronautics and Astronautics, University of Electronic Science and Technology of China, China. He received his bachelor degree from Zhoukou Normal University, China, in 2015. His research interests include human-robot system and exoskeleton robotics.

Qing Guo, received the B.E. degree in Automation from Harbin Institute of Technology, China, in 2003, and the Ph.D. degrees in Navigation, Guidance and Control from Harbin Institute of Technology, China, in 2008. Now Dr. Guo is a professor at School of Aeronautics and Astronautics, University of Electronic Science and Technology of China. His research interests include robust and adaptive control, electrohydraulics.

Huiyu Xiong, is currently a master candidate at Glasgow College, University of Electronic Science and Technology of China. His research interests include robust and adaptive control.

Dan Jiang received the B.E. degree in mechanical engineering and the M.S and Ph.D. degrees in fluid power transmission and control from Harbin Institute of Technology, China, in 2002, 2005, and 2009, respectively. Since 2009, she has been with School of Mechanical, Electronic and Industrial Engineering, University of Electronic Science and Technology of China, China, where she became a Lecturer. Since 2013, she has been an Associate Professor with the School of Mechanical, Electronic and Industrial Engineering. Her research interests include fluid transmission and control systems, pneumatic systems, microfluidic technology, and mechanical reliability.

Yao Yan, received the Ph.D. in Mechanics in 2014 from Tongji University, China. Now he is an Associate Professor at School of Aeronautics and Astronautics, University of Electronics Science and Technology of China. His research interests include regenerative cutting chatter and control for suppression, vibro-impact capsule robot, and exoskeleton robot.

\section{Funding}

Supported by National Natural Science Foundation of China (Grant Nos. 51775089, 12072068, 11872147), Sichuan Province Science and Technology Support Program of China (Grant Nos. 2020YFG0137, 2018JY0565).

\section{Competing Interests}

The authors declare no competing financial interests.

\section{Author Details}

${ }^{1}$ School of Aeronautics and Astronautics, University of Electronic Science and Technology of China, Sichuan 611731, Chengdu, China. ${ }^{2}$ Aircraft Swarm Intelligent Sensing and Cooperative Control Key Laboratory of Sichuan 
Province, Sichuan 611731, China. ${ }^{3}$ Glasgow College, University of Electronic Science and Technology of China, Sichuan 611731, China. ${ }^{4}$ School of Mechanical and Electrical Engineering, University of Electronic Science and Technology of China, Sichuan 611731, China.

Received: 4 February 2020 Revised: 12 November 2020 Accepted: 12 January 2021

Published online: 17 February 2021

\section{References}

[1] M Khamar, M Edrisi. Designing a backstepping sliding mode controller for an assistant human knee exoskeleton based on nonlinear disturbance observer. Mechatronics, 2018, 54:121-132.

[2] G Aguirre-Ollinger, J E Colgate, M A Peshkin, et al. Design of an active one-degree-of-freedom lower-limb exoskeleton with inertia compensation. The International Journal of Robotics Research, 2011, 30(4): 486-499.

[3] YYang, L Ma, D Huang. Development and repetitive learning control of lower limb exoskeleton driven by electrohydraulic actuators. IEEE Transactions on Industrial Electronics, 2017, 64(5): 4169-4178.

[4] X Li, Y H Liu, H Yu. Iterative learning impedance control for rehabilitation robots driven by series elastic actuators. Automatica, 2018, 90: 1-7.

[5] D Shi, W X Zhang, W Zhang, et al. A review on lower limb rehabilitation exoskeleton robots. Chinese Journal of Mechanical Engineering, 2019, 32(1): $1-11$.

[6] R Lu, Z Li, CY Su, et al. Development and learning control of a human limb with a rehabilitation exoskeleton. IEEE Transactions on Industrial Electronics, 2014, 61(7): 3776-3785.

[7] Q Guo, Y Zhang, D Jiang. A control approach for human-mechatronichydraulic-coupled exoskeleton in overload-carrying condition. International Journal of Robotics and Automation, 2016, 31 (4): 272-280.

[8] Q Guo, D Jiang. Method for walking gait identification in a lower extremity exoskeleton based on C4.5 decision tree algorithm. International Journal of Advanced Robotic Systems, 2015, 12.

[9] Z Li, CY Su, G Li, et al. Fuzzy approximation-based adaptive backstepping control of an exoskeleton for human upper limbs. IEEE Transactions on Fuzzy Systems, 2014, 23(3): 555-566.

[10] M K Dong, H Cheng, H T Tran, et al. Minimizing human-exoskeleton interaction force using compensation for dynamic uncertainty error with adaptive RBF network. Journal of Intelligent \& Robotic Systems, 2016, 82(34): 413-433.

[11] W He, Z Li, Y Dong, et al. Design and adaptive control for an upper limb robotic exoskeleton in presence of input saturation. IEEE Transactions on Neural Networks and Learning Systems, 2019, 30(1): 97-108.

[12] W Meng, Q Liu, Z Zhou, et al. Recent development of mechanisms and control strategies for robot-assisted lower limb rehabilitation. Mechatronics, 2015, 31: 132-145.

[13] N Hogan. Impedance control - an approach to manipulation. i - theory. ii - implementation. iii - applications. ASME Transactions Journal of Dynamic Systems \& Measurement Control B, 1985, 107: 1-24.
[14] A Q L Keemink, H van der Kooij, A H Stienen. Admittance control for physical human-robot interaction. The International Journal of Robotics Research, 2018, 37(11): 1421-1444.

[15] A N Sharkawy, P N Koustournpardis, N Aspragathos. Variable admittance control for human-robot collaboration based on online neural network training. 2018 IEEE/RSJ International Conference on Intelligent Robots and Systems (IROS), 2018: 1334-1339.

[16] J Buchli, F Stulp, ETheodorou, et al. Learning variable impedance control. The International Journal of Robotics Research, 2011, 30(7): 820-833.

[17] C Yang, G Peng, Y Li, et al. Neural networks enhanced adaptive admittance control of optimized robot-environment interaction. IEEE Transactions on Cybernetics, 2019, 49(7): 2568-2579.

[18] F Ferraguti, C T Landi, L Sabattini, et al. A variable admittance control strategy for stable physical human-robot interaction. The International Journal of Robotics Research, 2019, 38(6): 747-765.

[19] CT Landi, F Ferraguti, C Fantuzzi, et al. A passivity-based strategy for coaching in human-robot interaction. 2018 IEEE International Conference on Robotics and Automation (ICRA), 2018: 1-6.

[20] CT Landi, F Ferraguti, C Fantuzzi, et al. Admittance control parameter adaptation for physical human-robot interaction. 2017 IEEE International Conference on Robotics and Automation (ICRA), 2017: 2911-2916.

[21] F Dimeas, N Aspragathos. Online stability in human-robot cooperation with admittance control. IEEE Transactions on Haptics, 2016, 9(2): 267-278.

[22] D Ryu, J B Song, S Kang, et al. Frequency domain stability observer and active damping control for stable haptic interaction. IET Control Theory \& Applications, 2008, 2(4): 261-268.

[23] K Kronander, A Billard. Stability considerations for variable impedance control. IEEE Transactions on Robotics, 2016, 32(5): 1298-1305.

[24] M J Kim, W Lee, JY Choi, et al. A passivity-based nonlinear admittance control with application to powered upper-limb control under unknown environmental interactions. IEEE/ASME Transactions on Mechatronics, 2019, 24(4): 1473-1484.

[25] K Gui, H Liu, D Zhang, et al. A practical and adaptive method to achieve EMG-based torque estimation for a robotic exoskeleton. IEEE/ASME Transactions on Mechatronics, 2019, 24(2): 483-494.

[26] Y Zhuang, S Yao, C Ma, et al. Admittance control based on EMG-driven musculoskeletal model improves the human-robot synchronization. IEEE Transactions on Industrial Informatics, 2018, 15(2): 1211-1218.

[27] X Yu, W He, Y Li, et al. Bayesian estimation of human impedance and motion intention for human-robot collaboration. IEEE Transactions on Cybernetics, 2019. https://doi.org/10.1109/TCYB.2019.2940-276.

[28] P B G Dohmann, S Hirche. Distributed control for cooperative manipulation with event-triggered communication. IEEE Transactions on Robotics, 2020, 36(4): 1038-1052.

[29] K Ba, G Ma, B Yu, et al. A nonlinear model-based variable impedance parameters control for position-based impedance control system of hydraulic drive unit. International Journal of Control, Automation and Systems, 2020, 18(7): 1806-1817.

[30] S S Ge, T H Lee, C J Harris. Adaptive neural network control of robotic manipulators. World Scientific Publishing, Singapore, 1998.

\section{Submit your manuscript to a SpringerOpen ${ }^{\odot}$ journal and benefit from:}

- Convenient online submission

- Rigorous peer review

- Open access: articles freely available online

- High visibility within the field

Retaining the copyright to your article

Submit your next manuscript at $\boldsymbol{\nabla}$ springeropen.com 